Check for updates

Cite this: RSC Adv., 2018, 8, 35395

Received 3rd August 2018

Accepted 28th August 2018

DOI: $10.1039 / c 8 r a 06548 a$

rsc.li/rsc-advances

\title{
Synthesis, antitumor activity and molecular mechanism of doxorubicin conjugated trimethyl- chitosan polymeric micelle loading Beclin1 siRNA for drug-resisted bladder cancer therapy
}

\author{
Zhou Zhong, $\dagger^{\text {ac }}$ Zhong Cheng, $\dagger^{\mathrm{b}}$ Dongyuan Su, ${ }^{\mathrm{d}}$ Ting Xu, ${ }^{\mathrm{c}}$ Xiang $\mathrm{Li}^{\star \mathrm{a}}$ \\ and Fengbo Wu (iD)*ac
}

\begin{abstract}
Herein, we describe a convenient approach for the preparation of a polymeric micelle using doxorubicin (DOX) conjugated trimethyl-chitosan (TMC) with Beclin-1 siRNA (Si-Beclin1/DOX-TMC). This micelle displayed a potent capacity for autophagy inhibition and reversed drug-resistance to DOX in BIU-87/ADR cell lines. The Si-Beclin1/DOX-TMC micelle was highly cytotoxic to both drug-sensitive BIU-87 and drug-resistant BIU-87/ADR cells. Its capacity to reverse drug-resistance was dependent upon upregulation of autophagy levels in BIU-87/ADR cells. DOX was conjugated to TMC via a pH-sensitive Schiff base, which responded to the acidic lysosome microenvironment and resulted in the cytoplasmic release of DOX. The structure of DOX conjugation to the TMC polymeric micelle was characterized by NMR, GPC, TEM and DLS. DOX release profiles in different pH environment were determined by HPLC. Cellular uptake, changes to nuclei morphology and formation of autophagosomes were observed using a fluorescence microscope. Finally, in vivo antitumor activity of systemic Si-Beclin1/DOX-TMC micelle administration was evaluated in BIU-87/ADR xenograft models and Si-Beclin1/DOX-TMC micelles showed significantly suppressed tumor growth.
\end{abstract}

\section{Introduction}

Bladder cancer is one of the ten most common neoplasms worldwide, with an annual incidence of over 80000 new cases and an estimated 17000 deaths each year in the United States. ${ }^{1}$ In China, the estimated incidence and mortality due to bladder cancer are mostly due to common tumor of the urinary system. $^{2}$ Urothelial carcinoma (also called transitional cell carcinoma) is the predominant histopathologic subtype of bladder cancers. ${ }^{3-5}$ Bladder urothelial carcinoma is usually sensitive to combined chemotherapy, e.g. methotrexate, vinblastine, doxorubicin (DOX) and cisplatin (MVAC), or gemcitabine plus cisplatin (GC), which are standard therapies for advanced bladder cancer. ${ }^{6}$ However, over half of bladder cancer patients are likely to have a recurrence within five year

\footnotetext{
${ }^{a}$ Department of Urology and Department of Orthopaedic Surgery, West China Hospital, Sichuan University, Chengdu 610041, China.E-mail:xiangli.87@scu.edu.cn;wfb_sc@ 163.com

${ }^{b}$ Department of Gastrointestinal Surgery, West China Hospital, Sichuan University, Chengdu 610041, China

'Department of Pharmacy, West China Hospital, Sichuan University, Chengdu 610041, China

${ }^{d}$ Department of Gastroenterology, Chongzhou People's Hospital, Chengdu 611230, China

$\dagger$ These authors contributed equally.
}

of cystoscope cutting and chemotherapy. The main cause for the bladder cancer recurrence after chemotherapy is attributed to the presence of drug-resistant urothelial carcinoma cells, however, the detailed mechanisms involved in such drug-resistance and cancer recurrence are still unclear. ${ }^{7-9}$

Autophagy is an evolutionarily conserved biological process whereby intracellular organelles and compositions are encapsulated and degraded by autophagosomes and autolysosomes, respectively. ${ }^{10-12}$ Autophagy can be triggered by various extracellular signals and stresses including starvation, hypoxia, chemotherapeutic drugs, among others. Numerous studies have shown that autophagy acts as a double-edged sword that varies in different stages of tumor progression. ${ }^{13,14}$ In the carcinogenesis stage, autophagy has been shown to clean cells with harmful mutations or damaged organelles. In contrast, cancer cells within established tumors resist radiation and chemotherapy using autophagy. Regulation of autophagy-related genes by RNA interference has been reported to synergistically reverse multidrug resistance of cancer cells. Recently, Ojha et al. reported that drug-resistant bladder cancer cells have higher basal autophagic flux levels than that of sensitive ones. ${ }^{15-17}$ Chemotherapeutic treatment leads to increased levels of autophagy whereas the addition of autophagy inhibitors synergistically enhances cytotoxicity with such drugs on drug-resistant bladder cancer cells. ${ }^{18-22}$ 
Chitosan is a carbohydrate polymer comprised of $\mathrm{N}$-glucosamine or $\mathrm{N}$-acetylglucosamine. There are many reports on the applications of chitosan as drug or gene carrier because of its biocompatibility, low toxicity and versatility for chemical modifications. ${ }^{23}$ However, chitosan must be maintained in an aqueous solution of neutral or alkaline media, which remarkably limits its biomedical applications. Numerous studies have reported the preparation and biomedical properties of modified chitosan such as graft-copolymerization, alkylation or multiple alkylation, carboxymethylation, michael addition and direct conjugation to chemotherapeutics as well as peptides or proteins. $^{24-34}$ Chitosan derivatives, $\mathrm{N}$-quaternization modified chitosan, i.e. $\mathrm{N}, \mathrm{N}, \mathrm{N}$-trimethyl chitosan (TMC) achieves considerable attention as a drug and gene carrier because of its physiochemical stability, biocompatibility, synthetic feasibility and controllable cationic charge as well as its water-solubility via regulation of its hydrophilic and hydrophobic groups. ${ }^{35-38}$

Herein, we synthesized covalently conjugated DOX to TMC scaffold with siRNA Beclin-1 to form a polymeric micelle. We aimed to further find an efficient method for the establishment of a carbohydrate polymer-based drug/siRNA codelivery nanocarrier with both chemotherapeutic and autophagy inhibitory functions. Our co-delivery micelle was demonstrated to be effective against drug-resistance of bladder cancer cells in vitro and in vivo by regulating the balance between autophagy and apoptosis.

\section{Results and discussion}

\section{Synthesis of doxorubicin conjugated trimethyl chitosan (DOX- TMC)}

The synthetic process of DOX-TMC is shown in Fig. 1, and the molecular weight distributions of TMC and DOX-TMC were determined by GPC (gel permeation chromatography). As shown in Table $1, M_{\mathrm{n}}, M_{\mathrm{w}}$ and polydispersity index (PDI $=M_{\mathrm{w}}$ / $M_{\mathrm{n}}$ ) of DOX-TMC were $15 \mathrm{kDa}, 22 \mathrm{kDa}$ and 1.47, respectively. These results positively correlated to the molecular weight distribution of corresponding TMC or O-TMC. First, certain amine groups of the aminoglucose fragments in chitosan were quaternized by $\mathrm{CH}_{3} \mathrm{I}$ to form TMC. Then, aminoglucose fragments were oxidized to form dialdehyde by $\mathrm{KIO}_{4}$. DOX-TMC were synthesized by forming the Schiff's base between the amino groups of DOX and the aldehyde group of oxidized TMC.

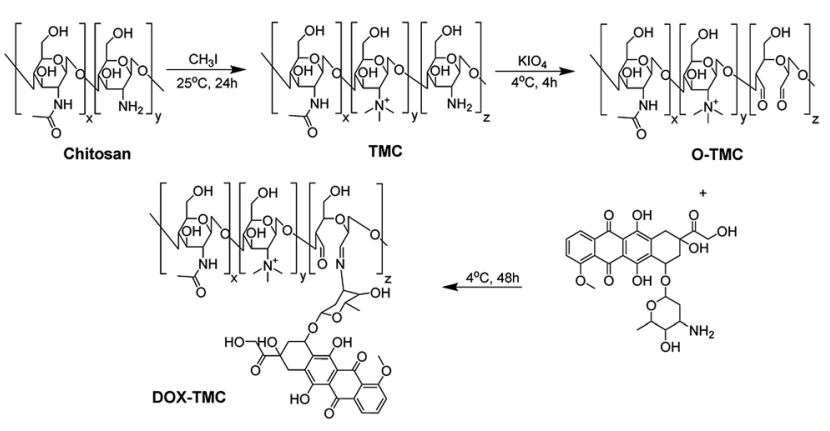

Fig. 1 Synthesis route of DOX-TMC.
Table 1 Molecular weights of various TMC derivatives

\begin{tabular}{llll}
\hline & $M_{\mathrm{n}}(\mathrm{kDa})$ & $M_{\mathrm{w}}(\mathrm{kDa})$ & PDI $\left(M_{\mathrm{w}} / M_{\mathrm{n}}\right)$ \\
\hline TMC & 29 & 51 & 1.75 \\
O-TMC & 11 & 17 & 1.54 \\
DOX-TMC & 15 & 22 & 1.47 \\
\hline
\end{tabular}

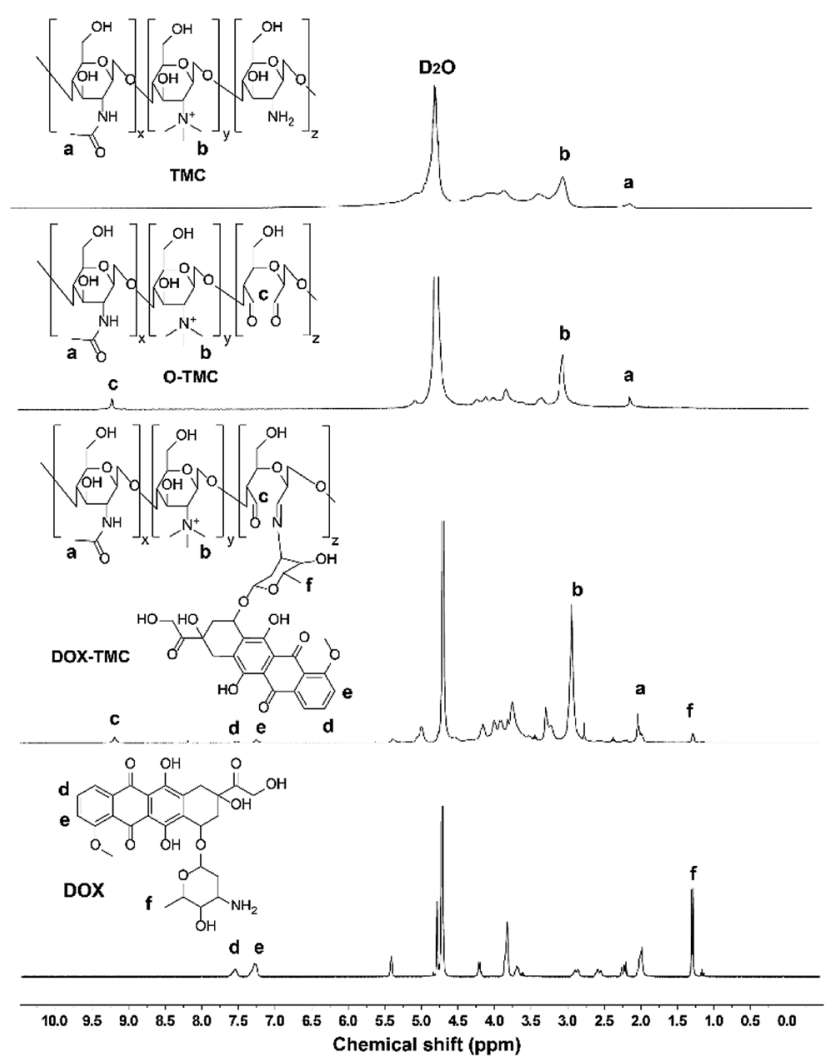

Fig. $2{ }^{1} \mathrm{H}$ NMR spectra of TMC, O-TMC, DOX and DOX-TMC.

The chemical structure of DOX-TMC was confirmed by ${ }^{1} \mathrm{H}$ NMR (Fig. 2). As reported in previous studies, a single peak at $2.1 \mathrm{ppm}$ was attributed to the hydrogen of acetyl group within TMC; signals at about $2.9 \mathrm{ppm}$ were attributed to the methyl hydrogen of the trimethylamine group within TMC. Additionally, the peaks at about 7.3 and $7.5 \mathrm{ppm}$ were attributed to the aromatic hydrogens of the anthracene group within DOX. The broad single peak around $9.1 \mathrm{ppm}$ was attributed to the aldehyde hydrogen of O-TMC and DOX-TMC. The multiple peaks between 2.8 and 4.0 were attributed to the oligosaccharide on TMC and DOX, respectively.

\section{Preparation and characterization of siRNA Beclin-1-loaded DOX-TMC polymeric micelle}

SiRNA Beclin-1-loaded DOX-TMC polymeric micelles were formed by electrostatic interactions between the positively charged TMC backbone and negatively charged siRNA. Fluctuations in particle size as well as the zeta potential of the micelles were plotted against the weight ratios of DOX-TMC/siRNA in Fig. 3. SiRNA-loaded DOX-TMC polymeric micelle particle size 
a

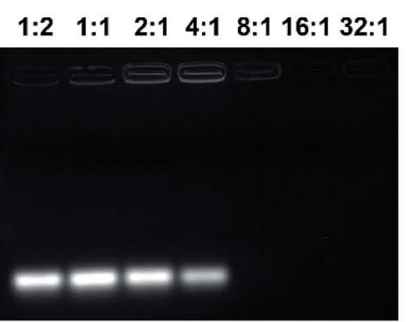

b

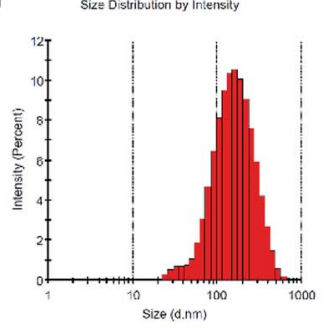

C

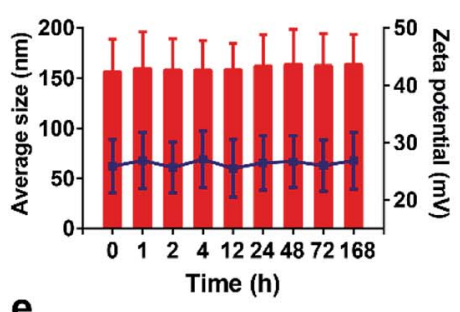

e

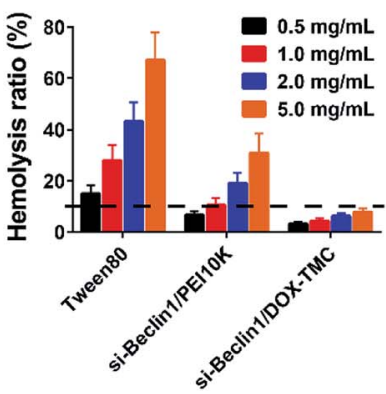

Fig. 3 Characterization of siRNA loaded DOX-TMC micelles. (a) Agarose gel electrophoresis of siRNA loaded by DOX-TMC; (b) the size distribution of siRNA loaded DOX-TMC micelles; (c) stability of siRNA loaded DOX-TMC micelles in 10\% serum; (d) transmission electron microscopy images of siRNA loaded DOX-TMC micelles (scale bar indicates $100 \mathrm{nM}$ ); (e) hemolysis test results different concentrations of siRNA loaded DOX-TMC micelles after incubation with red blood cells suspension for $3 \mathrm{~h}$.

increased with the ascending $\mathrm{N} / \mathrm{P}$ ratio from 0.5 to 16 in the range of 110 to $450 \mathrm{~nm}$. At an N/P ratio of 8.0, the micelles had the lowest mean particle size at $112.3 \mathrm{~nm}$. When the $\mathrm{N} / \mathrm{P}$ ratio increased from 0.5 to 16.0 , the zeta potential increased correspondingly, however, the zeta potential did not rise considerably when the N/P ratio was above 4.0.

Average particle size, size distribution and zeta potential of siRNA-loaded DOX-TMC polymeric micelles were determined by DLS (Dynamic Lighting Scattering) method using a Malvern ZS90 Zetasizer (Malvern Instruments Ltd., Malvern, UK) at room temperature. As shown in Fig. 3, mean micelle size was $152.3 \pm 30.4 \mathrm{~nm}$, with a PDI value of $0.237 \pm$ 0.19 (Fig. 3b), and a mean zeta potential of about $28.6 \pm$ $5.0 \mathrm{mV}$. The siRNA-loaded DOX-TMC polymeric micelle particle size was around $150 \mathrm{~nm}$ with a relatively narrow distribution, which provided the potential for accumulation at tumor sites via the EPR effect. Furthermore, the size distribution and zeta potentials of siRNA loaded DOX-TMC micelles displayed little change with incubation of $10 \%$ serum, suggesting the serum stability of DOX-TMC micelles (Fig. 3c). The morphology of siRNA-loaded DOX-TMC micelles were visualized with TEM. These micelles displayed a generally homogenous and nearly spherical morphology (Fig. 3d). The hemolysis tests also suggested the good biocompatibility of DOX-TMC micelles. There were less than $5 \%$ hemolysis caused by DOX-TMC micelles even at a concentration of $5.0 \mathrm{mg} \mathrm{mL}^{-1}$, which were far less than that of Tween 80 or PEI10K (Fig. 3e).

\section{In vitro evaluation of siRNA-loaded DOX-TMC polymeric micelles}

The release profiles of DOX from micelles behaved in a $\mathrm{pH}^{-}$ sensitive manner. Over $85 \%$ of free DOX molecules were released within 2 hours and almost all were released within 4 hours. In contrast, only about $40 \%$ of total DOX as part of DOXTMC micelles were released at $\mathrm{pH} 7.4$ and around $70 \%$ at $\mathrm{pH} 5.5$ (Fig. 4a), regardless of whether siRNA Beclin-1 was loaded into the micelles. These results suggest that siRNA-loaded DOX-TMC micelles could release DOX faster in an acidic microenvironment. In cytotoxicity assays, treating drug sensitive BIU-87 cells and resistant BIU87/ADR cells with blank TMC only caused marginal cytotoxicity (Fig. 4b), while treating these cells with siRNA Beclin-1 complex and TMC only slightly inhibited proliferation. Moreover, both free DOX and DOX-TMC displayed distinct cytotoxicity in BIU-87 cells but had nominal inhibitory effect on proliferation in BIU-87/ADR cells. Compared to free DOX, DOX-TMC caused higher cytotoxicity in BIU-87 cells, probably because the conjugation ability of TMC promoted cellular uptake. Finally, siRNA Beclin-1-loaded DOX-TMC micelles were observed to significantly inhibit cell proliferation in both BIU-87 and BIU-87/ADR cells.

\section{Intracellular distribution, autophagy and apoptosis induced} by siRNA-loaded DOX-TMC polymeric micelle

The cellular uptake and intracellular distribution of free DOX, DOX-TMC and FTIC-siRNA-loaded DOX-TMC micelles in BIU-
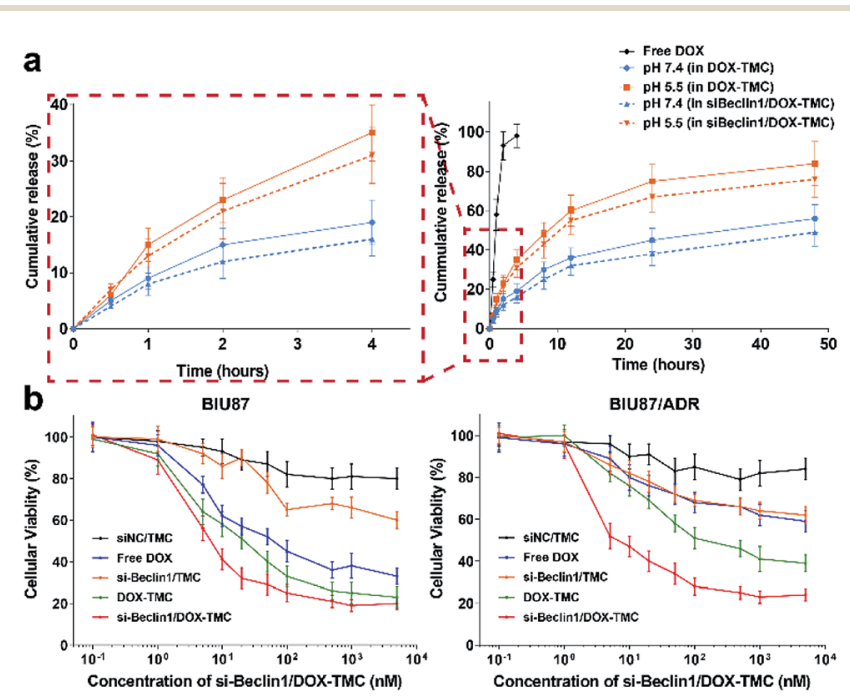

Fig. 4 (a) The accumulative release profiles of doxorubicin from DOXTMC and si-Beclin1/DOX-TMC in different pH environment; (b) cell viability curves of sensitive BIU87 cells and resistant BIU87/ADR cells incubated with siNC/TMC, si-Beclin1/TMC, free DOX, DOX-TMC and si-Beclin1/DOX-TMC, respectively. 
87/ADR cells were detected with a scanning confocal microscope. Both DOX and FITC-siRNA in siRNA-loaded DOX-TMC micelles were mostly taken up by free DOX or FITC-siRNA, respectively (Fig. 5a and b). Quantitative analysis indicated that the fluorescence intensity of FITC-siRNA, when delivered in DOX-TMC micelles, was 7.6-fold higher than when delivered as free siRNA. Fluorescence intensity of DOX when delivered in siRNA-loaded DOX-TMC micelles was 3.9-fold higher than when delivered as free DOX. Intracellular distribution analysis of FITC-siRNA and DOX in different groups demonstrated that siRNA was mainly localized to the cytoplasm and DOX was generally distributed in both the cytoplasm and nuclei.

Basal autophagy levels of BIU-87 and BIU-87/ADR cells were detected after transfection with the GFP-CL3 plasmid. As shown in Fig. 5c, the average puncta in BIU-87/ADR cells were remarkably higher than that of BIU-87 cells. With free DOX treatment, the average autophagy levels in both BIU-87 and BIU87/ADR cells were significantly boosted. As expected, the GFPLC3 puncta induced by DOX treatment in BIU-87/ADR cells were significantly more than that of BIU-87 cells.

In order to suppress the drug resistance related autophagy in BIU-87/ADR cells, siRNA Beclin-1 was delivered using TMC or DOX-TMC as non-viral carriers. As shown in Fig. $5 c$, both basal autophagy and DOX-induced autophagy were remarkably declined. For further study of the relationship between autophagy and apoptosis in drug-resistant bladder cancer cells, apoptosis by BIU-87/ADR cells induced by free DOX, siRNA Beclin-1, DOX-TMC and siRNA Beclin-1-loaded DOX-TMC were analysed by flow cytometry with Annexin V/PI dual staining and nuclei morphological changes with Hoechst-33258 staining (Fig. 6). We observed that si-Beclin1/TMC, free DOX and DOX-

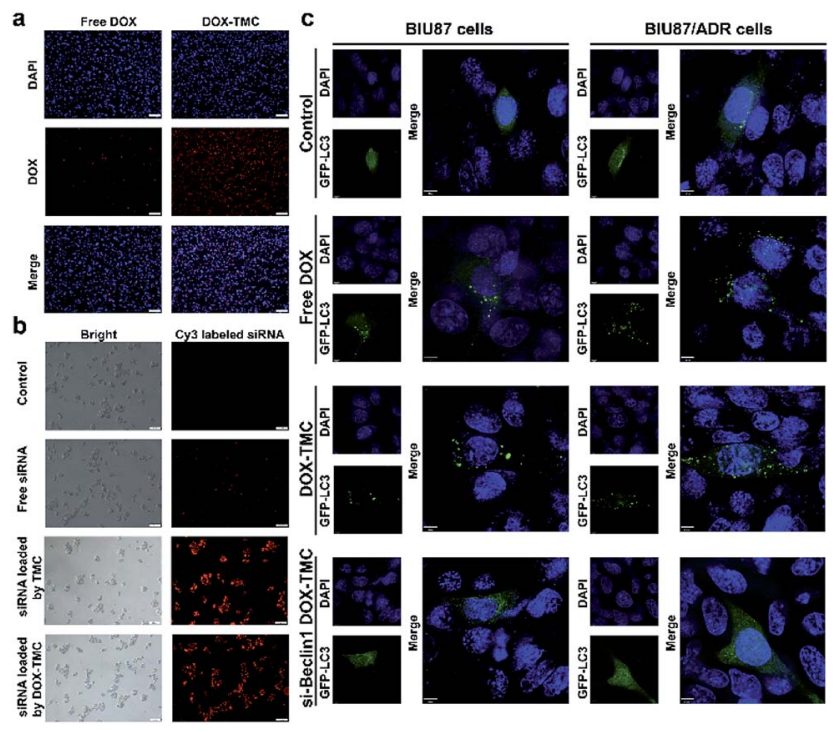

Fig. 5 (a) The cellular uptake of doxorubicin of free DOX or DOX-TMC by incubated with BIU87/ADR cells for $24 \mathrm{~h}$ (scale bar indicates 200 $\mu \mathrm{M})$; (b) cellular uptake of Cy3-labeled siRNA of free siRNA, complexed by TMC or complexed by DOX-TMC after incubation with BIU87/ADR cells for $24 \mathrm{~h}$ (scale bar indicates $20 \mu \mathrm{M}$ ); (c) observation of GFP-LC3 puncta stimulated by free DOX, DOX-TMC and si-Beclin1/DOX-TMC in BIU87 or BIU87/ADR cells (scale bar indicates $6 \mu \mathrm{M}$ ).

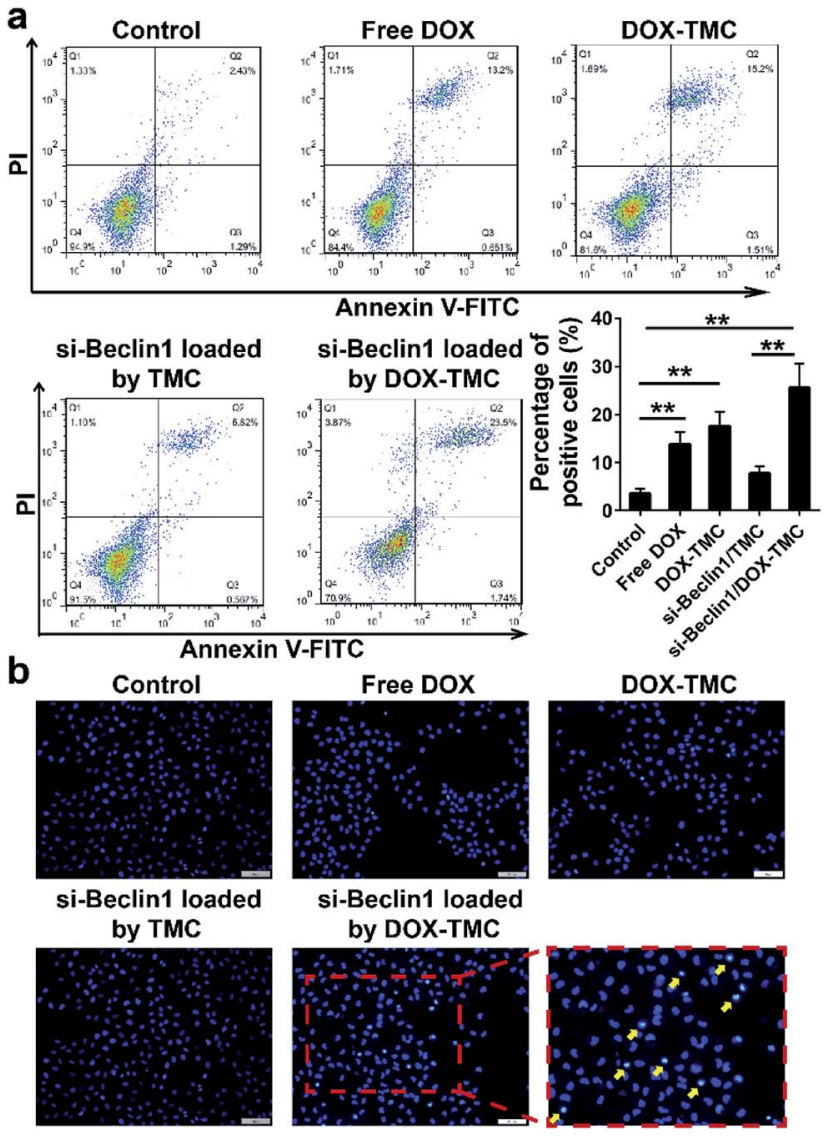

Fig. 6 The apoptosis assays of BIU87/ADR cells treated by free DOX, DOX-TMC, si-Beclin1/TMC and si-Beclin1/DOX-TMC, respectively; (a) flow cytometry assay stained by Annexin V/PI dual-staining; (b) the nuclei morphological changes stained by Hochest33258 (scale bar indicates $50 \mu \mathrm{M}$; ** $p<0.01$ ).

TMC only induced marginal to moderate apoptosis in BIU-87/ ADR cells, respectively. The siRNA Beclin-1-loaded DOX-TMC notably boosted apoptosis in drug-resistant BIU-87/ADR cells. These results suggested that the addition of siRNA Beclin-1 may overcome the autophagy-related resistance of BIU-87/ADR cells and synergistically enhance the cytotoxicity of DOX. Beclin-1 is one of the most important subunits of the PI3K complex III (PI3KC3), which plays a vital role in the initiation of the autophagosome. Deficiency in Beclin-1 directly inhibits the formation of autophagosome, which reverses the autophagydependent drug resistance of BIU-87/ADR cells.

SiRNA Beclin-1 synergistically suppresses proliferation and reverses drug-resistance in BIU-87/ADR cells by inhibiting autophagy and inducing mitochondrial apoptosis

To identify the mechanism by which the autophagy and apoptosis and their interactions via siBeclin-1/DOX-TMC are responsible for its anti-cancer effects, we treated BIU-87/ADR cell lines with siBeclin-1/DOX-TMC alone or siBeclin1/DOXTMC together with the autophagy inhibitor 3-MA or apoptosis inhibitor Z-VAD-FMK, and then assessed the cell proliferation ratio by MTT assays and apoptosis level with Annexin V-FITC/PI 
a

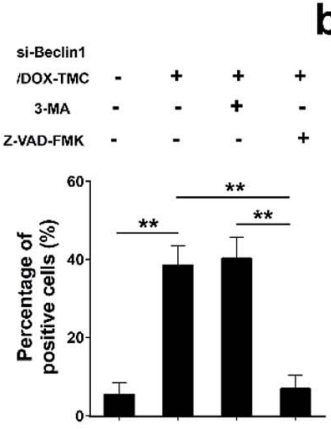

C
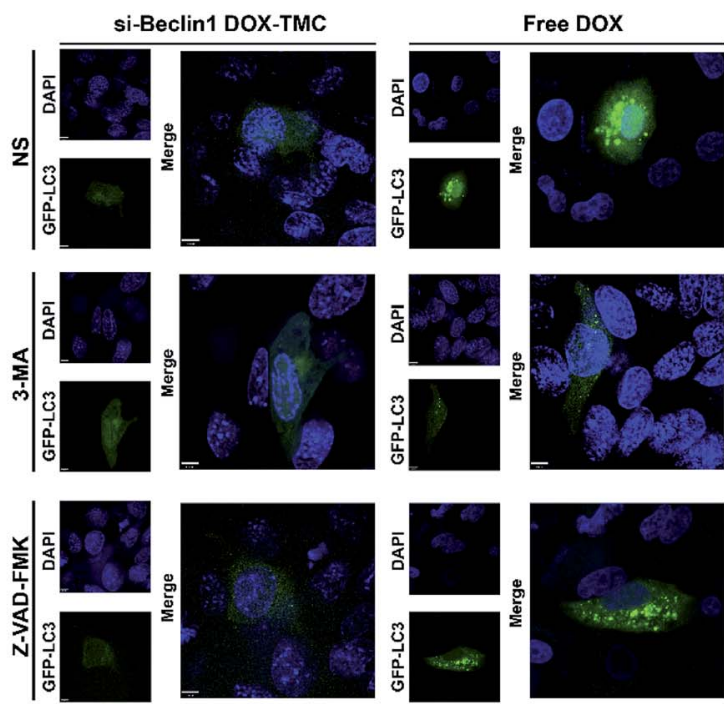

Fig. 7 (a) The changes on total apoptosis levels following si-Beclin1/ DOX-TMC combined with autophagy inhibitor 3-MA or apoptosis inhibitor Z-VAD-FMK; (b) the changes on autophagy or apoptosis related proteins following si-Beclin1/DOX-TMC combined with 3-MA or Z-VAD-FMK; (c) the changes on autophagy levels followed by siBeclin1/DOX-TMC or free DOX combined with 3-MA or Z-VAD-FMK (scale bar indicates $6 \mu \mathrm{M} ; * * p<0.01$ ).

staining followed by flow cytometry. The apoptotic BIU-87/ADR cells induced by siBeclin-1/DOX-TMC could be reversed by 3-MA or Z-VAD-FMK (Fig. 7a and b), respectively. Similarly, western blotting showed that both 3-MA and Z-VAD-FMK suppressed the cleavage of caspase-9 and PARP induced by siBeclin1/DOXTMC, but the siBeclin-1/DOX-TMC promoted SQSTM1 degradation was only reversed by 3-MA rather than Z-VAD-FMK (Fig. $7 b)$. These results suggested that siBeclin-1/DOX-TMC induced BIU-87/ADR cell apoptosis depended on inhibition of protective autophagy.

\section{siBeclin-1/DOX-TMC polymeric micelle suppresses drug- resistant bladder cancer cells in xenograft models}

In vivo anti-tumor effects of siBeclin-1/DOX-TMC micelles and its potential molecular mechanisms were validated in a BIU87/ADR bladder cancer xenograft model. The siBeclin-1/DOXTMC micelles displayed a higher tumor inhibition capacity than free DOX, DOX-TMC and siBeclin-1 lipoplex (Fig. 8a). The greatest tumor proliferation ratio (T/C) of the siBeclin-1/DOXTMC micelles was $36.7 \%$. The results based on tumor volume

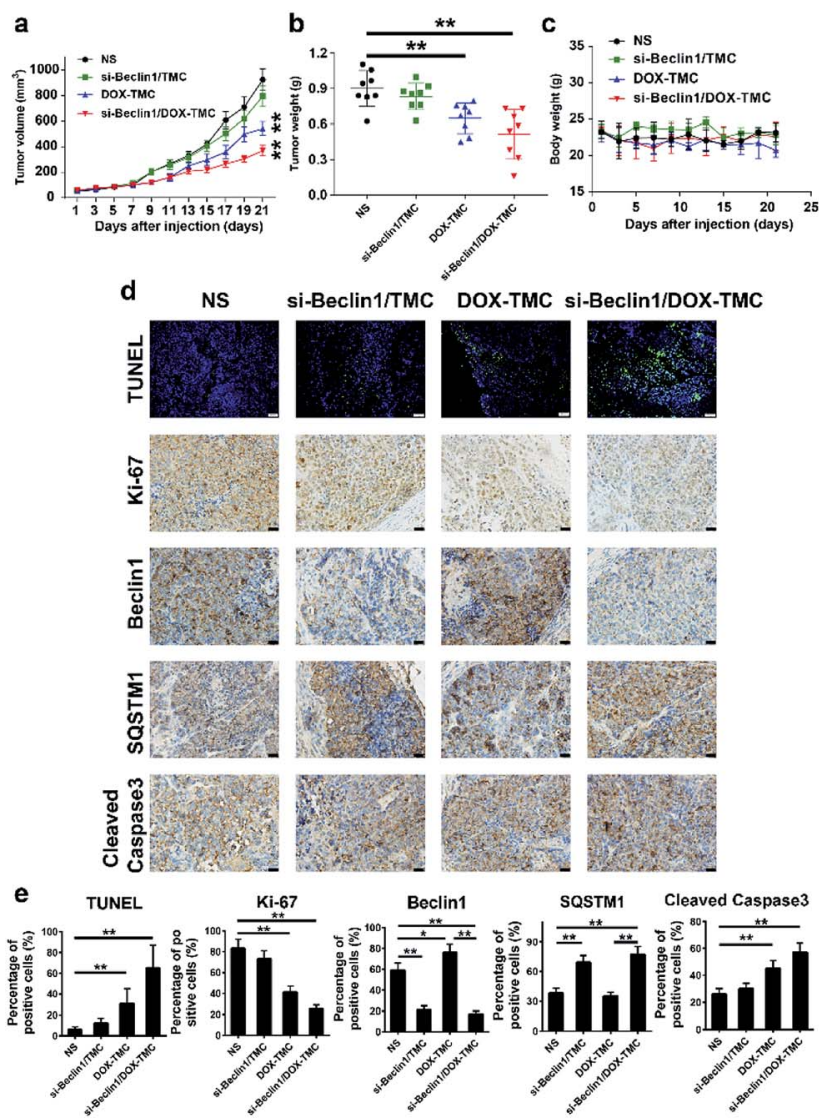

Fig. 8 Antitumor effects of si-Beclin1/DOX-TMC in vivo, compared to effects observed with NS, si-Beclin1/TMC and DOX-TMC. (a) curves of tumor volumes in each group; (b) statistics of tumor weight in each group; (c) curves of body weight changes; (d) immunohistological staining against TUNEL (scale bar indicates $50 \mu \mathrm{M}$ ), Ki67, Beclin1, SQSTM1 and cleaved caspase3 (scale bar indicates $20 \mu \mathrm{M}$ ); (e) quantification of each marker in panel $C\left({ }^{*} p<0.05\right.$ to NS group, ${ }^{* *} p<0.01$ to NS group, respectively).

changes were also confirmed by evaluation of excised tumor weights (Fig. 8b). The IHC (immunohistochemistry) analysis demonstrated that both siBeclin-1/DOX-TMC micelles and siBeclin-1 lipoplex knocked down the expression of Beclin-1 and up-regulated SQSTM1 expression, but the apoptosis marker cleaved caspase-3 was only activated in siBeclin-1/ DOX-TMC micelles group (Fig. 8c-e). Treatment of tumorbearing mice with free DOX or DOX-TMC only showed marginal therapeutic effect, partially because of the protective autophagy induced by DOX in BIU-87/ADR cells. We also assessed the changes of apoptosis markers TUNEL and proliferation marker Ki-67 in all groups (Fig. 8e), they were consistent with measurements of tumor volume and weight. These results indicate that DOX induced protective autophagy either as free drug or conjugated form, which could be reversed by co-delivery with siBeclin-1. In brief, siBeclin-1 suppressed protective autophagy in BIU-87/ADR cells and reversed their drug resistance. These in vitro and in vivo results may better describe the mechanism by which the combination of siBeclin-1 and DOX-TMC achieves the synergistic antitumor activity. 


\section{Experimental}

\section{Materials and regents}

Chitosan (with the acetylation degree of $10 \%$ ) was purchased from Sigma-Aldrich Chemical Co. (Burlington, MA, USA). Doxorubicin hydrochloride was purchased from Energy Chem. Co. (Shanghai, China). The other chemicals obtained were of analytical grade and directly used. BIU87 and BIU87/ ADR cells were obtained from National Infrastructure of Cell Line Resource (Wuhan, China) and maintained in Dulbecco's Modified Eagle's Medium containing 10\% fetal bovine serum and $1 \%$ penicillin-streptomycin (Invitrogen, Waltham, USA) in $5 \% \mathrm{CO}_{2}$ at $37^{\circ} \mathrm{C}$.

\section{Synthesis and characterization of DOX-TMC}

The experimental procedures to synthesize O-methylation free TMC is depicted in Fig. 1 and is according to the method reported by Muzzarelli, Verheul and our previous studies, with some modifications. ${ }^{35,39,40}$ In brief, chitosan was suspended in the mixture of formic acid and $37 \%$ formaldehyde; after refluxing for 12 hours the solvent was removed by vacuum distillation. The residue was neutralized by $1 \mathrm{~mol} \mathrm{~L}^{-1} \mathrm{NaOH}$ solution, then filtered and washed by deionized water. The iodomethane and NMP ( $N$-methylpiperidine) were added and the reaction mixture was kept at room temperature for 24 hours. The reaction mixture was poured into ethanol/diethyl ether $(\mathrm{v} / \mathrm{v}$ $=50: 50$ ), and the precipitated TMC was collected by centrifugation and washed twice by diethyl ether. The TMC was oxidized according to the procedure reported by Vold et al. and Bao et al. with few modifications. ${ }^{\mathbf{4 1 , 4 2}}$ The potassium periodate was added into acetate buffer solution of TMC under nitrogen atmosphere at $4{ }^{\circ} \mathrm{C}$. The reaction was performed for 4 hours at $4{ }^{\circ} \mathrm{C}$ and quenched by $10 \%$ ethylene glycol. The oxidative TMC (O-TMC) was purified by dialysis (MWCO 3500) and then lyophilization. DOX-TMC was prepared by coupling the aldehyde groups of O-TMC with the primary amine groups of doxorubicin. The TMC dissolved in deionized water was added into aqueous solution of doxorubicin, the reaction mixture was kept at $4{ }^{\circ} \mathrm{C}$ for $48 \mathrm{~h}$ in the dark. The crude product was purified by dialysis to remove unreacted doxorubicin and then lyophilized to obtain the final product DOX-TMC.

The chemical structures of TMC, O-TMC and DOX-TMC were determined by ${ }^{1} \mathrm{H}$ NMR at $400 \mathrm{MHz}$ (AvanceIII, Bruker, Germany) by using $\mathrm{D}_{2} \mathrm{O}$ as solvent. The relative molecular weight and molecular weight distributions of TMC, O-TMC and DOXTMC were determined by GPC method (gel permeation chromatography, USA) by a Waters 510 instrument and Waters 2410 refractive index detector.

\section{Preparation and characterization of SiRNA/DOX-TMC complexes}

The siRNA/DOX-TMC complexes were prepared in various $\mathrm{w} / \mathrm{w}$ (weight/weight) ratios of DOX-TMC to siRNA. In brief, both siRNA and DOX-TMC were dissolved in HEPES buffer $(20 \mathrm{mM}$, pH 7.4), the DOX-TMC stock solutions were added to the siRNA solution with expected proportions, the siRNA/DOX-TMC complexes were prepared by vortex-mixing and self-assembly. The hydrodynamic diameter and zeta potential of siRNA/DOXTMC micelles were measured using dynamic light scattering (DLS) (Zetasizer Nano ZS90, Malvern, UK) at room temperature while the morphology of siRNA/DOX-TMC micelles was further analysed by TEM (Hitachi, JEM-2000, Japan). The RNA binding capacity of DOX-TMC was analysed by gel electrophoresis, as described in our previous reports. ${ }^{43}$ The haemolysis ability of DOX-TMC was evaluated by the method described in previously published procedure. ${ }^{18}$ The DTX release profiles from siRNA/ DOX-TMC micelles were investigated using dialysis method as described before. ${ }^{35}$

\section{In vitro cellular toxicity analysis}

The cell viability assay was performed by a MTT assay; BIU87 cells and BIU87/ADR cells were plated in $1 \times 10^{4}$ cells per well into 96-well plates and incubated overnight at $37{ }^{\circ} \mathrm{C}$. Then, the cells were treated with free drugs or micelles at various concentrations for $24 \mathrm{~h}$. The MTT assay was measured at $570 \mathrm{~nm}$ by a Thermo Fisher Multiskan MK3 microplate reader (Thermo Fisher Scientific, MA, USA).

\section{In vitro intracellular uptake and distribution}

To observe the intracellular uptake and distribution of micelles, BIU87/ADR cells were plated onto a glass Petri dish in $1 \times 10^{4}$ cells per $\mathrm{cm}^{2}$ overnight. Subsequently, cells were incubated with culture medium containing free DOX, DOX-TMC, free cy3SiRNA, cy3-siRNA/TMC and cy3-siRNA/DOX-TMC (DOX $10 \mathrm{ng}$ $\mathrm{mL}^{-1}$, cy3-siRNA $100 \mathrm{nM}$ ). After incubating for another 2 hours, cells were rinsed with PBS, and then fixed with $4 \%$ paraformaldehyde. The nuclei were stained with DAPI $\left(4^{\prime}, 6-\right.$ diamidino-2-phenylindole) and observed by fluorescence microscopy (Olympus FV-1000, Olympus, Japan).

\section{Apoptosis and autophagy assays}

The cell apoptosis-inducing effects of micelles in BIU87/ADR cells were determined using flow cytometry. BIU87/ADR cells were seeded in six-well plates under $5 \% \mathrm{CO}_{2}$ at $37{ }^{\circ} \mathrm{C}$, and then co-cultured with free DOX, DOX-TMC, siBeclin-1/TMC and siBeclin-1/DOX-TMC, respectively (10 nM DOX and $100 \mathrm{nM}$ siBeclin-1) at desired $\mathrm{pH}$ value for $24 \mathrm{~h}$. Cells were stained with Annexin V-APC and propidium iodide (PI), then analyzed by flow cytometry. Untreated cells were used as negative controls. For autophagy assay, BIU87 and BIU87/ADR cells were transfected with GFP-LC3 plasmid, then added with free DTX, free DOX, DOX-TMC and siBeclin-1/DOX-TMC, respectively for $48 \mathrm{~h}$. The formation of autophagosome puncta were observed by confocal laser scanning microscopy (CLSM) (Leica TCS SP2, Lecia microsystems, German). Untreated groups were used as negative controls.

\section{Western blot analysis}

BIU87/ADR cells were plated into six-well plates and incubated overnight, then treated with siBeclin1/DOX-TMC with or without 3-MA, Z-VAD-FMK. The cells were harvested and re- 
suspended on ice for 10 min with RIPA lysis buffer, followed by centrifugation for $5 \mathrm{~min}$. The following steps for western blot analysis is described in our previous reports. ${ }^{35}$

\section{Xenograft models and immunohistochemistry analysis}

The in vivo antitumor activity of siBeclin1/DOX-TMC were performed according to the Guidelines for the Care and Use of Laboratory Animals (National Standard of the P. R. China, no. GB/T 35982-2018), and study procedures were approved by the Committee on Ethics of Animal Experimentation of West China Hospital of Sichuan University. The BIU87/ADR tumor-bearing mice were obtained by subcutaneous injection of BIU87/ADR cells $\left(6 \times 10^{5}\right.$ cells per mice) into the right forelimb region of male BALB/C nude mice. When the tumors grew to about 100 $\mathrm{mm}^{3}$ in volume, the mice were randomly divided into four groups with eight mice in each group as follows: NS, free DOX, DOX-TMC and siBeclin1/DOX-TMC. The mice were intravenously injected at the equivalent dose of $5 \mathrm{mg} \mathrm{kg}{ }^{-1}$ DTX and $5 \mathrm{mg} \mathrm{kg}^{-1}$ siBeclin1. The tumor volume and body weight of mice were monitored every two days for 21 days. After the mice were euthanized, a $4 \mu \mathrm{m}$ section was cut from tumor tissues. All sections were fixed in $4 \%$ paraformaldehyde overnight and subjected to paraffin embedding. Immunostaining for Ki-67, Beclin1, SQSTM1 and cleaved caspase 3 were performed according to standard protocols. The primary antibody was an anti-mouse monoclonal antibody against Ki-67, Beclin1, SQSTM1 and cleaved caspase3, respectively. The sections were incubated with the primary antibody at RT for 2-4 hours and then washed with the secondary antibody for $30 \mathrm{~min}$, the reaction was finally visualized using a VECTASTAIN ABC Kit and detected with diaminobenzidine (DAB) as the chromogen. The immunofluorescent staining of TUNEL were performed according to our previously reported method. ${ }^{35}$

\section{Conclusions}

In the current study, we report a convenient approach to prepare a polymeric micelle based on doxorubicin conjugated trimethyl-chitosan with Beclin-1 siRNA. The prepared SiBeclin1/DOX-TMC micelle silences the Beclin-1 protein expression to inhibit protective autophagy of BIU-87/ADR cells, while simultaneously inducing apoptosis. Our study presents a novel strategy to overcome chemotherapy resistance by nanomedicines, in which regulating the autophagy and apoptosis are integrated by rational design and preparation of multifunctional biocompatible carriers of siRNA and drug codelivery. These results indicate that co-delivery of Beclin-1 siRNA and DOX by a biocompatible carbohydrate based nanocarrier can effectively suppress drug-resistant bladder cancer in preclinical studies.

\section{Conflicts of interest}

There are no conflicts to declare.

\section{Acknowledgements}

We are grateful for financial support from the National Natural Science Foundation of China (81672552 and 81603065), the Science \& Technology Department of Sichuan Province (2017JY0226) and the 1.3.5 project for disciplines of excellence, West China Hospital, Sichuan University (ZY2016104).

\section{Notes and references}

1 R. L. Siegel, K. D. Miller and A. Jemal, CA, 2018, 68, 7-30.

2 W. Chen, R. Zheng, P. D. Baade, S. Zhang, H. Zeng, F. Bray, A. Jemal, X. Q. Yu and J. He, CA, 2016, 66, 115-132.

3 Y. Du, L. Wang, W. Wang, T. Guo, M. Zhang, P. Zhang, Y. Zhang, K. Wu, A. Li, X. Wang, J. He and J. Fan, J. Biomed. Nanotechnol., 2018, 14, 161-167.

4 S. Antoni, J. Ferlay, I. Soerjomataram, A. Znaor, A. Jemal and F. Bray, Eur. Urol., 2017, 71, 96-108.

5 S. Chavan, F. Bray, J. Lortet-Tieulent, M. Goodman and A. Jemal, Eur. Urol., 2014, 66, 59-73.

6 S. K. Sweeney, Y. Luo, M. A. O'Donnell and J. G. Assouline, J. Biomed. Nanotechnol., 2017, 13, 232-242.

7 M. Katoh and H. Nakagama, Med. Res. Rev., 2014, 34, 280300.

8 M. A. Tabrizi, P. G. Baraldi, S. Baraldi, S. Gessi, S. Merighi and P. A. Borea, Med. Res. Rev., 2017, 37, 936-983.

9 J. W. Tessmann, J. Buss, K. R. Begnini, L. M. Berneira, F. R. Paula, C. M. P. de Pereira, T. Collares and F. K. Seixas, Biomed. Pharmacother., 2017, 94, 37-46.

10 Z. P. Pan, Y. J. Chen, J. Y. Liu, Q. L. Jiang, S. Y. Yang, L. Guo and G. He, Eur. J. Med. Chem., 2018, 144, 517-528.

11 N. Zhang, Y. Huang, F. B. Wu, Y. B. Zhao, X. Li, P. F. Shen, L. Yang, Y. Luo, L. Yang and G. He, Mol. Pharmaceutics, 2016, 13, 2466-2483.

12 B. W. Ke, M. Tian, J. J. Li, B. Liu and G. He, Med. Res. Rev., 2016, 36, 983-1035.

13 K. A. Bauckman and I. U. Mysorekar, Autophagy, 2016, 12, 850-863.

14 Y. G. Liang, J. L. Zhu, H. S. Huang, D. M. Xiang, Y. Li, D. Y. Zhang, J. X. Li, Y. L. Wang, H. L. Jin, G. S. Jiang, Z. Y. Liu and C. S. Huang, Autophagy, 2016, 12, 1229-1239.

15 M. Ishaq, R. Ojha, K. Sharma, G. Sharma, S. K. Singh and S. Majumdar, Biochim. Biophys. Acta, Mol. Cell Res., 2016, 1863, 2560-2573.

16 R. Ojha, V. Jha and S. K. Singh, Bba-Mol Cell Res, 2016, 1863, 347-359.

17 R. Ojha, S. K. Singh and S. Bhattacharyya, Biochim. Biophys. Acta, Gen. Subj., 2016, 1860, 2484-2497.

18 L. L. Chen, M. Qian, L. W. Zhang, J. Xia, Y. M. Bao, J. Y. Wang, L. Y. Guo and Y. C. Li, $R S C A d v$., 2018, 8, 17710-17722.

19 Y. G. Chen, X. X. Li, H. Xiao, J. P. Xiao, B. Li, X. Y. Chen, Y. Wang, D. Cheng and X. T. Shuai, RSC Adv., 2018, 8, 2082-2091.

20 N. Xu, X. B. Huang, G. F. Yin, M. J. Bu, X. M. Pu, X. C. Chen, X. M. Liao and Z. B. Huang, RSC Adv., 2018, 8, 15604-15612. 
21 J. L. Zhang, Y. Luo, X. F. Zhao, X. W. Li, K. X. Li, D. W. Chen, M. X. Qiao, H. Y. Hu and X. L. Zhao, RSC Adv., 2016, 6, 113173-113184.

22 M. Zhang, X. Zhao, Z. Fang, Y. Niu, J. Lou, Y. Wu, S. Zou, S. Xia, M. Sun and F. Du, RSC Adv., 2017, 7, 3369-3375.

23 M. Amidi, E. Mastrobattista, W. Jiskoot and W. E. Hennink, Adv. Drug Delivery Rev., 2010, 62, 59-82.

24 Y. Lu, X. Y. Li, X. D. Zhou, Q. Wang, X. W. Shi, Y. M. Du, H. B. Deng and L. B. Jiang, RSC Adv., 2014, 4, 33355-33361.

25 F. Y. Ding, H. B. Deng, Y. M. Du, X. W. Shi and Q. Wang, Nanoscale, 2014, 6, 9477-9493.

26 X. Q. Zhang, H. Zhang, L. Q. Yin, R. Hu, T. Qiu, Y. H. Yin, X. Xiong, H. Zheng and Q. Wang, J. Biomed. Nanotechnol., 2016, 12, 1688-1698.

27 S. J. Xin, X. Y. Li, Q. Wang, R. Huang, X. L. Xu, Z. J. Lei and H. B. Deng, J. Biomed. Nanotechnol., 2014, 10, 803-810.

28 R. Hu, H. Zheng, J. Cao, Z. Davoudi and Q. Wang, J. Biomed. Nanotechnol., 2017, 13, 1097-1105.

29 R. Hu, H. Zheng, J. Cao, Z. Davoudi and Q. Wang, J. Biomed. Nanotechnol., 2017, 13, 1058-1068.

30 W. Li, X. Y. Li, Q. Wang, Y. J. Pan, T. Wang, H. G. Wang, R. Song and H. B. Deng, Carbohydr. Polym., 2014, 99, 218225.

31 Y. Yang, S. P. Wang, Y. T. Wang, X. H. Wang, Q. Wang and M. W. Chen, Biotechnol. Adv., 2014, 32, 1301-1316.

32 Y. W. Cai, C. F. Wu, Z. Y. Liu, L. J. Zhang, L. H. Chen, J. Q. Wang, X. K. Wang, S. T. Yang and S. Wang, Environ. Sci.: Nano, 2017, 4, 1876-1886.
33 D. Q. Chen, C. Wang, F. Jiang, Z. Liu, C. Y. Shu and L. J. Wan, J. Mater. Chem. B, 2014, 2, 4726-4732.

34 Y. X. Wang, X. Niu, X. L. Guo, H. Yu, Z. H. Liu, Z. Q. Zhang and S. Yuan, Glycobiology, 2018, 28, 318-332.

35 B. Li, Q. Ma, G. He, X. R. Song, F. B. Wu, Y. Zheng, S. Zeng, C. Liu and W. Ren, Colloid Polym. Sci., 2013, 291, 1319-1327.

36 Y. Zheng, X. R. Song, G. He, Z. Cai, Y. Zhou, B. Yu, J. P. Xu, Y. Q. Wei and S. X. Hou, J. Drug Targeting, 2011, 19, 647-656.

37 B. Xu, Q. S. Jin, J. Zeng, T. Yu, Y. Chen, S. Z. Li, D. Q. Gong, L. L. He, X. Y. Tan, L. Yang, G. He, J. H. Wu and X. R. Song, ACS Appl. Mater. Interfaces, 2016, 8, 25753-25769.

38 Y. B. Zhao, D. Y. Lin, F. B. Wu, L. Guo, G. He, L. Ouyang, X. R. Song, W. Huang and X. Li, Int. J. Mol. Sci., 2014, 15, 17565-17576.

39 R. A. A. Muzzarelli and F. Tanfani, Carbohydr. Polym., 1985, 5, 297-307.

40 R. J. Verheul, M. Amidi, S. van der Wal, E. van Riet, W. Jiskoot and W. E. Hennink, Biomaterials, 2008, 29, 3642-3649.

41 I. M. Vold and B. E. Christensen, Carbohydr. Res., 2005, 340, 679-684.

42 X. Bao, W. Wang, C. Wang, Y. Wang, J. Zhou, Y. Ding, X. Wang and Y. Jin, Biomaterials, 2014, 35, 8450-8466.

43 X. Wang, F. Wu, G. Li, N. Zhang, X. Song, Y. Zheng, C. Gong, B. Han and G. He, Acta Biomater., 2018, 74, 414-429. 